\title{
Dossier Engagement public des chercheurs Introduction. De la République des savants à la démocratie technique : conditions et transformations de l'engagement public des chercheurs
}

\author{
Christophe Bonneuil
}

L'histoire et la sociologie des intellectuels conçoivent l'intellectuel comme « un homme ou une femme qui applique à l'ordre politique une notoriété acquise ailleurs » (Julliard et Winock, 1996, p. 12), et l'on peut alors définir l'engagement des chercheurs comme mise en jeu dans l'espace public d'une parole dont l'autorité est liée à sa position dans le champ scientifique ${ }^{1}$. Mais l'engagement public d'un scientifique ne va pas de soi aujourd'hui. Prendre parti publiquement sur un problème, n'est-ce pas déroger à la neutralité scientifique, transgresser la frontière entre science et politique? Voire - dans le cas de la mise en public d'enjeux des sciences et des techniques - commettre l'erreur de soumettre aux profanes des questions complexes que seuls les «collègues compétents » peuvent convenablement traiter? En écho à cette réticence des chercheurs, certains analystes se sont alors demandé si le chercheur en sciences dites «dures » est encore un intellectuel ou si la figure de l'intellectuel n'a pas, peu à peu, depuis 1945, cédé la place dans l'espace public à celles de $1^{\prime}$ « expert », du « technocrate » (Habermas, 1973 ; Pinault, 2003) ou du technicien, simple rouage peu réflexif d'une «science normale ${ }^{2}$ » devenue "technoscience ». Il est vrai que, même au cours de la mobilisation "Sauvons la recherche» de 2004-2006, la défense d'une recherche statutaire autonome et moins « pilotée » fut moins argumentée au nom des apports à la vie démocratique et sociale d'une autonomie préservée des chercheurs et enseignants-chercheurs publics qu'au nom des retombées économiques de la recherche et de la préservation de son «attractivité ».

\footnotetext{
${ }^{1}$ Dans les différentes contributions de ce dossier, on traitera de l'engagement des chercheurs lorsqu'il s'exprime dans la sphère publique, et non pas au sein des seuls cercles scientifiques et de l'expertise.

${ }^{2}$ Sur la notion de science normale, voir Kuhn (1983).
}

En écho à cette situation, les sciences humaines et sociales n'ont accordé qu'une attention restreinte à l'engagement des scientifiques. C'est un chantier peu exploré par l'histoire des intellectuels (Sirinelli, 1986; Leymarie et Sirinelli, 2003), essentiellement centrée sur des figures de la littérature ou des sciences humaines (Zola, Gide, Sartre, Bourdieu...), mais aussi par l'histoire des sciences ou par la sociologie (Pinault, 2003). Cette faible présence de l'engagement des chercheurs dans l'espace public, cette faible reconnaissance de leur rôle dans la vie démocratique, expliquent peut-être également l'inexistence de dispositifs spécifiques de protection de la liberté d'expression et d'alerte des chercheurs en France, alors que plusieurs pays développés ont adopté des lois de protection des lanceurs d'alerte ${ }^{3}$.

Si nous abordons ici la question de l'engagement public des scientifiques, c'est parce qu'il nous semble que l'analyse (et la protection) de l'engagement des chercheurs constitue un nœud privilégié du nécessaire dialogue entre sciences et sociétés, au même titre que l'analyse (et l'appui) des démarches de production de connaissances par des groupes citoyens. Les figures et les formes prises par l'engagement public des scientifiques depuis un demi-siècle sont multiples. Quitte à schématiser, nous en proposons une périodisation qui permet de les penser au sein des transformations des modes de production et de régulation des savoirs scientifiques (Pestre, 2003) et des transformations de l'espace public de la science et de l'expertise depuis 50 ans (Bonneuil, 2005). Trois périodes peuvent être distinguées.

\footnotetext{
${ }^{3}$ Voir, dans ce numéro, l'article de Christine Noiville et Marie-Angèle Hermitte, "Quelques pistes pour un statut juridique du chercheur lanceur d'alerte».
} 
1. De l'affaire Dreyfus (Duclert, 1999) au colloque de Caen (1956) : on a affaire à une forme d'engagement complexe, "fait, d'une part, de défense organisée de la communauté scientifique [...] assimilée à la défense de l'intérêt de la République, de l'État, et passant par la conquête de positions de pouvoir, d'autre part, de promotion de la science - identifiée au progrès moral, intellectuel et social - et de mobilisation de $1^{\prime \prime \prime}$ esprit scientifique" au service d'une intervention critique des savants dans la sphère publique » (Pinault, 2003, p. 246). La référence à l'affaire Dreyfus comme mythe fondateur de la vocation politique des scientifiques est d'ailleurs souvent réactivée après 1945. Ainsi, pour Paul Langevin (1964), il est "nécessaire que la science tende la main à la justice, par l'application des méthodes scientifiques à l'étude des problèmes humains et par un développement de la conscience civique chez ceux qui contribuent au développement de la science. À l'exemple des intellectuels qui, au temps de l'affaire Dreyfus, mirent leur force d'esprit au service de la justice individuelle, c'est aujourd'hui un devoir, pour ceux qui créent la science, de veiller à l'usage qu'en font les hommes ». De la création de l'Union rationaliste en 1930 à l'appel de Stockholm - origine du Mouvement de la Paix - lancé notamment par Frédéric Joliot-Curie, l'engagement des chercheurs relève d'un rapport pédagogique à la société qu'il s'agit d'instruire. On retrouve encore ce modèle chez Jacques Monod, lorsqu'il défend l'avortement au nom d'une définition scientifique de la personne humaine qui commencerait avec l'apparition du système nerveux central ${ }^{4}$; ou bien dans la posture faussement modeste du physicien Alfred Kastler - animateur des comités Vietnam avec le mathématicien Laurent Schwartz -, qui légitime l'intervention des scientifiques dans le champ politique par leur rapport privilégié à l'objectivité : "La compétence que nous, scientifiques, possédons dans le domaine étroit qui est le nôtre ne saurait en aucun cas nous qualifier pour guider les hommes dans d'autres domaines de l'activité humaine, en particulier dans le domaine social et politique. [...] Le service principal que nous pouvons rendre à nos concitoyens est d'essayer d'aborder les problèmes avec l'objectivité qui caractérise les discussions scientifiques, d'essayer de dépassionner le débat ${ }^{5}$. »

2. Si ce modèle du scientifique éducateur et représentant de l'Universel (ou de l'objectivité) reste prégnant jusqu'à aujourd'hui, Mai 1968 annonce l'émergence d'un deuxième modèle d'engagement intellectuel des chercheurs, questionnant leur champ de savoir dans ses implications sociales et politiques, politisant les tensions

\footnotetext{
${ }^{4}$ Voir, dans ce numéro, l'article de Jean-Paul Gaudillière, «Intellectuels engagés et experts : biologistes et médecins dans la bataille de l'avortement ».

${ }^{5}$ Kastler, A., 1970. Ce que les savants apportent au monde, c'est leur objectivité, Le Figaro littéraire, 1-7 juin.
}

traversées par leur milieu et revendiquant une science «pour le peuple» (Jaubert et Lévy-Leblond, 1973). D'une part, la linéarité de la relation entre science, croissance et bien-être ne fait plus l'unanimité (Bonneuil, 2005; Boy, 1999). La CFDT parle des "dégâts du progrès", tandis que se développe dans les milieux politicointellectuels une critique de la technocratie, du pouvoir de la technique, du scientisme, du projet des Lumières, de la raison instrumentale (Mumford, Ellul, Marcuse, Habermas, Foucault, Illich, etc.). D'autre part, la politisation estudiantine des années 1960 a produit un militantisme de type nouveau chez les jeunes chercheurs et universitaires, dont un nombre important adhère au gauchisme et enlève pour quelque temps au parti communiste la majorité du Syndicat national des chercheurs scientifiques et du Syndicat national de l'enseignement supérieur (SNESUP). Les clivages de positionnements lors des événements de 1968 (Brillant, 2003) ne font que s'ajouter aux tensions liées à une division du travail qui a accompagné le développement massif de la recherche pendant les trente glorieuses, générant des conflits sociaux sans précédent dans les laboratoires (grèves, comme au laboratoire Leprince-Ringuet en 1969, luttes anti-hiérarchiques, revues Labo-Contestation ou Le Cri des labos, etc.). Dans ce double contexte, l'engagement du chercheur ne peut plus se parer aussi facilement d'une image homogène et consensuelle de la science comme productrice d'un point de vue supérieur sur le monde. À la figure du «savant engagé », une nouvelle génération oppose alors la figure du «chercheur responsable», qui politise son champ de compétence. Cette politisation est plus ou moins radicale. Elle prend des formes diverses, qui vont de l'alerte de spécialistes sur les impacts sociétaux d'un domaine de recherche ou $\mathrm{d}^{\prime}$ une décision technologique ${ }^{6}$ à un travail plus continu de contre-information ou de contre-expertise «au service» des mouvements sociaux, assorti de la recherche de futurs sociotechniques alternatifs. Deux contributions à ce numéro sont de bons exemples de cette dernière démarche : le Groupe Information Santé évoqué par J.-P. Gaudillière et le Groupement scientifique d'information sur l'énergie nucléaire (GSIEN) étudié par S. Topçu ${ }^{7}$. Cette prise de position peut aller jusqu'au rejet de la posture d'expert, fût-il «contre-expert», considérée comme dépossession d'autres paroles de la base, et à l'invention de dispositifs d'enquête permettant au contraire de produire un savoir à partir de la parole d'acteurs

\footnotetext{
${ }^{6}$ L'appel Berg sur le génie génétique ou l'Appel des 400 contre le programme électronucléaire en 1975 en sont de bons exemples. Ils prolongent le travail passé des atomistes responsables de Pugwash.

7 Mais on peut aussi citer, parmi d'autres, le collectif intersyndical Amiante de Jussieu : voir Moll-François (2005).
} 
non académiques ${ }^{8}$. Le type de positionnement d'une nébuleuse dense de collectifs de "chercheurs critiques" au sein de ce spectre résulte de trois facteurs principaux. Le premier est générationnel : ceux qui n'ont pas connu l'affaire Lyssenko et le mot d'ordre de la «science prolétarienne » sont moins réticents à concevoir la science comme une "pratique sociale parmi d'autres ; irrémédiablement marquée par la société où elle s'insère, elle en porte tous les traits et en reflète toutes les contradictions » et à dénoncer $1^{\prime}$ «illusion de l'autonomie des activités intellectuelles » (Jaubert et Lévy-Leblond, 1973, p. 17). Le second est lié à l'ancrage politique : maoïsme ou anarchosyndicalisme se prêtent mieux à des postures de critique de «l'idéologie de la compétence » et du scientisme que le communisme PC et le trotskisme. Le troisième, enfin, est la position occupée dans un champ scientifique où chaque domaine présente une épistémologie spontanée et une identité un peu différente : un physicien ne critique pas sa discipline de la même façon qu'un psychiatre et, à l'intérieur de la physique, les frontières d'identité entre « appliqué » et « fondamental » ne sont pas sans effets sur les postures d'engagement.

Malgré la diversité des postures d'engagement à la croisée de ces trois paramètres, on peut estimer que cette nouvelle génération de chercheurs engagés rompt avec le modèle d'engagement des savants comme «intellectuel universel », et se rapproche d'une posture d' « intellectuel spécifique ». Cette catégorie d' «intellectuel spécifique» est forgée par Foucault pour théoriser la pratique des intellectuels (y compris scientifiques et techniciens) de la gauche radicale, dont il fait partie, comme une pratique de lutte dans son propre milieu et son secteur particulier et de politisation de son propre champ de savoir dans l'espace public (Foucault, 1994).

3. Mais, dans un contexte de reflux global des mobilisations et de l'engagement à partir de la fin des années 1970, cette figure décline à son tour alors que s'ouvre une troisième période. La critique radicale de la science par les chercheurs cède le pas autour de 1981 à la prise de responsabilités institutionnelles, la reconversion vers les sciences sociales (notamment l'histoire et la sociologie des sciences qui s'institutionnalisent) ou l'implication dans la promotion de la "culture scientifique et technique » (Bonneuil, 2005; Petitjean, 1998; Debailly, 2005). Au point que l'engagement dans des organisations ou des luttes liées à la responsabilité sociale des chercheurs est aujourd'hui résiduel dans le milieu scientifique, a fortiori chez les moins de 50 ans. La thématique de la "responsabilité sociale des chercheurs » a même largement disparu des discours publics sur la science de ces dernières années. On voit alors, à la fin des années 1980 et dans les années 1990, la figure du «lanceur

\footnotetext{
8 Par exemple, le Groupe Information Prison (1971-1972) et la revue Impascience.
}

d'alerte » (Chateauraynaud et Torny, 1999) supplanter celle, déclinante, du "chercheur responsable » comme intellectuel spécifique. Plus individuelle, focalisée sur des risques physiques plutôt que s'affichant directement comme une critique sociale des institutions scientifiques, cette figure n'est nullement récente, mais elle est devenue autrement plus saillante depuis les crises sanitaires et environnementales des vingt dernières années (Cicolella et Benoit-Browaeys, 2005). Hormis ces lanceurs d'alerte peu nombreux, ce sont désormais plus souvent des organisations d'une société civile plus éduquée et plus distante du credo du progrès (associations de malades, de solidarité, écologistes, altermondialistes...) que des collectifs de chercheurs qui mettent en politique les enjeux des sciences et des techniques et polarisent l'espace quelque peu rétréci - de l'engagement des chercheurs. En témoignent la comparaison entre le GSIEN des années 1970 et l'ACRO ou la CRIIRAD d'après Tchernobyl $1^{9}$ ou entre le Groupe d'information biologie de 1975 et l'engagement des chercheurs dans la controverse OGM ces dernières années ${ }^{10}$. La mise en critique de la science, qui était minoritaire, mais active, entre 1968 et 1981 au sein des milieux scientifiques, émane essentiellement, depuis la fin des années 1980, de l'extérieur de celle-ci.

Cette nouvelle configuration appelle alors de nouvelles réflexions, à la croisée de la question de l'engagement des chercheurs et de la recherche d'un nouveau contrat entre science et société : en quoi la prise de parole des chercheurs au-delà du cercle des pairs est-elle une nécessité démocratique? Quelles en sont les conditions de possibilité, mais aussi les risques? Quel rôle accorder à l'alerte dans la «société du risque » et dans notre «modernité réflexive» (Beck, 2001) ? Et comment protéger les lanceurs d'alerte et un droit à l'expression des chercheurs pour garantir une qualité démocratique du débat sur les différents futurs sociotechniques et les mondes communs qui restent possibles ${ }^{11}$ ? Après l'échec des Boutiques de Sciences en France au début des années 1980, peut-on constituer des «coproductions » entre recherche publique et acteurs sociétaux à but non lucratif du «tiers secteur de la connaissance » (Callon, 1998; Fondation Sciences citoyennes, 2004 ; FutuRIS, 2004) ? Et, plus largement, au-delà de la défense d'un statut et de moyens, et au-delà des «alertes », y a-t-il encore une place pour un engagement de chercheurs en faveur d'une gouvernance démocratique des choix technoscientifiques face au seul pilotage des marchés - et des biens communs de connaissance - face au durcissement de la propriété intellectuelle?

\footnotetext{
9 Voir, dans ce numéro, l'article de Sezin Topçu, « Nucléaire : de l'engagement "savant" aux contre-expertises associatives». 10 Voir, dans ce numéro, l'article de Christophe Bonneuil, «Cultures épistémiques et engagement public des chercheurs dans la controverse OGM ».

${ }^{11}$ Cf. supra, note 3.
} 


\section{Références}

Beck, U., 2001. La Société du risque : sur la voie d'une autre modernité, Paris, Aubier.

Bonneuil, C., 2005. Les transformations des rapports entre sciences et société en France depuis la Seconde Guerre mondiale : un essai de synthèse, in Le Marec, J., Babou, I. (Eds), Actes du colloque Sciences, médias et société, Lyon, ENSLSH, 15-40 (http://sciences-medias.ens-lsh.fr/IMG/pdf/ actes.pdf).

Boy, D., 1999. Le Progrès en procès, Paris, Presses de la Renaissance.

Brillant, B., 2003. Les Clercs de 68, Paris, PUF.

Callon, M., 1998. Des différentes formes de démocratie technique, Annales des Mines / Responsabilité E Environnement, 9, 63-73.

Chateauraynaud, F., Torny, D., 1999. Les Sombres Précurseurs : une sociologie pragmatique de l'alerte et du risque, Paris, EHESS.

Cicolella, A., Benoit-Browaeys, D., 2005. Alertes santé : experts et citoyens face aux intérêts privés, Paris, Fayard.

Debailly, R. 2005. La Science en procès : formes et évolutions de la critique radicale de la science en France. Mémoire de DEA en sciences sociales et philosophie de la connaissance, Université Paris IV.

Duclert, V., 1999. L'engagement scientifique et l'intellectuel démocratique. Le sens de l'affaire Dreyfus, Politix, 48, 71-94.

Fondation Sciences citoyennes, 2004. Quelle politique scientifique pour entrer dans le $21^{\mathrm{e}}$ siècle? Vers un nouveau contrat entre recherche et société. Contribution aux états généraux sur l'avenir de la recherche, note $\mathrm{n}^{\circ} 2$ (http://sciencescitoyennes.org/article.php3?id_article=1440).
Foucault, M., 1994. La fonction politique de l'intellectuel [1976], in Defert, D., Ewald, F. (Eds), Dits et écrits, Paris, Gallimard, III, 109-114.

FutuRIS, 2004. Socialiser l'innovation, un pari pour demain (http://www.operation-futuris.org/images/ rapport_citoyennete.pdf).

Habermas, J., 1973. La Technique et la science comme idéologie, Paris, Gallimard.

Julliard, J, Winock, M., 1996. Dictionnaire des intellectuels français, Paris, Le Seuil.

Jaubert, A., Lévy-Leblond, J.-M., 1973. Autocritique de la science, Paris, Le Seuil.

Kuhn, T., 1983 [1 $1^{\text {re }}$ éd. angl. 1962]. La Structure des révolutions scientifiques, Paris, Flammarion.

Langevin, P., 1964. Discours lors de l'hommage solennel du Front national universitaire, Grand amphithéâtre de la Sorbonne, 3 mars 1945, in Langevin, P., La Pensée et l'action, Paris, Éditions sociales, 308-310.

Leymarie, M., Sirinelli, J.-F. (Eds), 2003. L'Histoire des intellectuels aujourd'hui, Paris, PUF.

Moll-François, F., 2005. La Construction du risque amiante en France. Mémoire de DEA, EHESS, Paris.

Pestre, D., 2003. Science, argent et politique, Paris, INRA Éditions.

Petitjean, P., 1998. La critique des sciences en France, Alliage, 35-36, 118-133.

Pinault, M., 2003. L'intellectuel scientifique : du savant à l'expert, in Leymarie, M., Sirinelli, J.-F. (Eds), L'Histoire des intellectuels aujourd'hui, Paris, PUF, 229-254.

Sirinelli, J.-F., 1986. Le hasard ou la nécessité? Une histoire en chantier : 1'histoire des intellectuels, Vingtième siècle. Revue d'histoire, 9, 97-107.

To access this journal online: www.edpsciences.org 